TRANSACTIONS OF THE

AMERICAN MATHEMATICAL SOCIETY

Volume 356, Number 2, Pages 735-755

S 0002-9947(03)03298-7

Article electronically published on September 22, 2003

\title{
SYMMETRIZATION, SYMMETRIC STABLE PROCESSES, AND RIESZ CAPACITIES
}

\author{
DIMITRIOS BETSAKOS
}

Dedicated to Albert Baernstein on the occasion of the thirty years of his star-function

\begin{abstract}
Let $\mathrm{X}_{t}$ be a symmetric $\alpha$-stable process killed on exiting an open subset $D$ of $\mathbb{R}^{n}$. We prove a theorem that describes the behavior of its transition probabilities under polarization. We show that this result implies that the probability of hitting a given set $B$ in the complement of $D$ in the first exit moment from $D$ increases when $D$ and $B$ are polarized. It can also lead to symmetrization theorems for hitting probabilities, Green functions, and Riesz capacities. One such theorem is the following: Among all compact sets $K$ in $\mathbb{R}^{n}$ with given volume, the balls have the least $\alpha$-capacity $(0<\alpha<2)$.
\end{abstract}

\section{INTRODUCTION AND STATEMENTS OF THE RESULTS}

The M.Riesz kernels in $\mathbb{R}^{n}, n \geq 2$, are the functions

$$
k_{\alpha}(x)=\frac{\mathcal{A}(n, \alpha)}{|x|^{n-\alpha}}, \quad x \in \mathbb{R}^{n} \backslash\{0\},
$$

where $\alpha \in(0, n)$ and

$$
\mathcal{A}(n, \gamma)=\frac{\Gamma\left(\frac{n-\gamma}{2}\right)}{\Gamma\left(\left|\frac{\gamma}{2}\right|\right) 2^{\gamma} \pi^{n / 2}}, \gamma \in \mathbb{R} .
$$

These kernels include as special and limiting cases the kernels of classical potential theory: the Newtonian and logarithmic kernels. If $K$ is a compact set in $\mathbb{R}^{n}$ and $\mu$ is a Borel probability measure on $K$, the $\alpha$-energy of $\mu$ is

$$
I_{\alpha}(\mu)=\int_{K} \int_{K} k_{\alpha}(x-y) d \mu(x) d \mu(y) .
$$

The $\alpha$-capacity of $K$ is defined by

$$
C_{\alpha}(K)=\left(\inf _{\mu} I_{\alpha}(\mu)\right)^{-1},
$$

where the infimum is taken over all Borel probability measures on $K$. When $n \geq 3$, the 2-capacity of $K$ is the Newtonian capacity. We will denote by $m_{n}(K)$ the $n$-dimensional Lebesgue measure (volume) of $K \subset \mathbb{R}^{n}$. A classical isoperimetrictype result conjectured by H.Poincaré in 1903 and proved by G.Szegö in 1930 [27], 20, pp. 63-65] states that among all compact sets in $\mathbb{R}^{n}, n \geq 3$, with the same

Received by the editors July 14, 2002 and, in revised form, January 23, 2003.

2000 Mathematics Subject Classification. Primary 31B15, 60J45.

Key words and phrases. Symmetrization, symmetric stable process, polarization, transition function, $\alpha$-harmonic measure, Green function, Riesz capacity. 
volume, the balls have minimal Newtonian capacity. An analogous result holds for logarithmic capacity in the plane. It is an open problem to extend these results for other values of $\alpha$. This problem is implicitly stated by P.Mattila in [19, p.192]. We will prove the following:

Theorem 1. Let $\alpha \in(0,2)$. Let $K$ be a compact set in $\mathbb{R}^{n}$ with $m_{n}(K)=V>0$. Let $\mathbb{B}_{V}$ be a ball in $\mathbb{R}^{n}$ with $m_{n}\left(\mathbb{B}_{V}\right)=V$. Then

$$
C_{\alpha}(K) \geq C_{\alpha}\left(\mathbb{B}_{V}\right) \text {. }
$$

If $n \geq 3$ and $\alpha \in(2, n)$, the problem remains open.

Theorem 1 will be obtained by the method of symmetrization. A modern approach to symmetrization is via another class of geometric transformations, called polarizations.

The polarization $E^{*}$ of set $E \subset \mathbb{R}^{n}$ is defined as follows: Let

$$
\Pi_{n-1}=\left\{\left(x_{1}, x_{2}, \ldots, x_{n}\right) \in \mathbb{R}^{n}: x_{n}=0\right\} .
$$

For $A \subset \mathbb{R}^{n}$, we denote by $\widehat{A}$ the reflection of $A$ in the $(n-1)$-dimensional plane $\Pi_{n-1}$. Thus we have

$$
\widehat{A}=\left\{\left(x_{1}, \ldots, x_{n-1}, x_{n}\right):\left(x_{1}, \ldots, x_{n-1},-x_{n}\right) \in A\right\} .
$$

We will also use the following notation:

$$
\begin{aligned}
& \text { if } x=\left(x_{1}, \ldots, x_{n-1}, x_{n}\right) \text { then } \hat{x}:=\left(x_{1}, \ldots, x_{n-1},-x_{n}\right) ; \\
& A_{+}:=\left\{\left(x_{1}, \ldots, x_{n-1}, x_{n}\right) \in A: x_{n}>0\right\} \\
& A_{-}=\left\{\left(x_{1}, \ldots, x_{n-1}, x_{n}\right) \in A: x_{n}<0\right\} .
\end{aligned}
$$

Now, let $E$ be any set in $\mathbb{R}^{n}$. We divide $E$ into three subsets $S, U, V$ :

$$
S=\{x \in E: \hat{x} \in E\}=E \cap \widehat{E}
$$

(the "symmetric part" of $E$ ),

$$
U=\left\{x \in E: x \in E_{+}, \hat{x} \notin E\right\}
$$

(the "upper non-symmetric part" of $E$ ),

$$
V=\left\{x \in E: x \in E_{-}, \hat{x} \notin E\right\}
$$

(the "lower non-symmetric part" of $E$ ).

Then $E=S \cup U \cup V$. The polarization $E^{*}$ of $E$ is the set

$$
E^{*}:=S \cup U \cup \widehat{V} \text {. }
$$

If $x \in \mathbb{R}^{n}$, we set

$$
x^{*}=\left\{\begin{array}{l}
x, \text { if } x \in \mathbb{R}_{+}^{n} \cup \Pi_{n-1}, \\
\hat{x}, \text { if } x \in \mathbb{R}_{-}^{n} .
\end{array}\right.
$$

It is clear that the polarizarion of an open set is open. The polarization as defined above may be called polarization with respect to $\Pi_{n-1}$. In a similar way, one can define polarization with respect to any other oriented $(n-1)$-dimensional plane in $\mathbb{R}^{n}$.

By an ingenious method due to V.Wolontis [28], various types of symmetrization can be approximated by a sequence of polarizations. We refer to [1], [3], [8], [14], [15], 25] for the history, main properties, and various applications of polarization in complex function theory, potential theory, differential equations, and Brownian motion. 


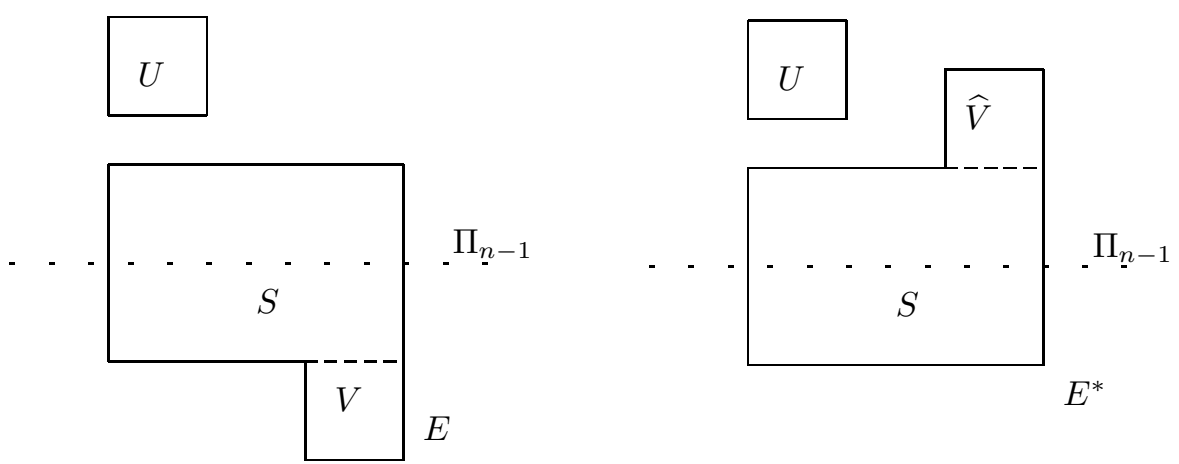

Figure 1. A set $E$ and its polarization $E^{*}$.

Theorem 2. Let $\alpha \in(0,2)$, and let $K$ be a compact set in $\mathbb{R}^{n}, n \geq 2$. Then

$$
C_{\alpha}(K) \geq C_{\alpha}\left(K^{*}\right) .
$$

For $\alpha=2$, this result follows from the work of Wolontis [28] $(n=2)$ and V.N.Dubinin [14] $(n \geq 3)$. A well-known procedure leads from Theorem 2 to Theorem 1. We will describe this procedure briefly in Section 4. The proof of Theorem 2 is based on some aspects of the potential theory of Riesz kernels. We will now review these aspects.

Definition 1. Let $\alpha \in(0,2)$, and let $D$ be an open set in $\mathbb{R}^{n}, n \geq 2$. A function $u: \mathbb{R}^{n} \rightarrow \mathbb{R}$ is called $\alpha$-harmonic in $D$ if

(a) $u$ is continuous in $D$;

(b) $u$ is in $\mathcal{L}^{1}$, that is,

$$
\int_{|x|>1} \frac{|u(x)|}{|x|^{n+\alpha}} d x<\infty ;
$$

(c) for every ball $B\left(x_{o}, r\right)$ with closure in $D$,

$$
u\left(x_{o}\right)=\int_{\mathbb{R}^{n}} u(x) \varepsilon_{\alpha}^{(r)}\left(x-x_{o}\right) d x,
$$

where

$$
\varepsilon_{\alpha}^{(r)}(x)= \begin{cases}\frac{\Gamma(n / 2) \sin (\pi \alpha / 2) r^{\alpha}}{\pi^{n / 2+1}\left(|x|^{2}-r^{2}\right)^{\alpha / 2}|x|^{n}}, & |x|>r, \\ 0, & |x|<r .\end{cases}
$$

Definition 2. Let $\alpha \in(0,2)$ and $f \in \mathcal{L}^{1}$. For $\varepsilon>0$ and $x \in \mathbb{R}^{n}$, we define

$$
\Delta_{\varepsilon}^{\alpha / 2} f(x)=\mathcal{A}(n,-\alpha) \int_{|y-x|>\varepsilon} \frac{f(y)-f(x)}{|y-x|^{n+\alpha}} d y
$$

and

$$
\Delta^{\alpha / 2} f(x)=\lim _{\varepsilon \downarrow 0} \Delta_{\varepsilon}^{\alpha / 2} f(x),
$$

whenever the limit exists. 
By [7] Theorem 3.9], a function $u$ defined on $\mathbb{R}^{n}$ is $\alpha$-harmonic in an open set $D$ if and only if it is continuous in $D$ and $\Delta^{\alpha / 2} u=0$ in $D$.

Suppose now that $D$ is an open bounded set in $\mathbb{R}^{n}$ having boundary which satisfies an exterior cone condition, i.e., there is a cone $\mathcal{C} \subset \mathbb{R}^{n}$ such that for every $\zeta \in \partial D$ there is a cone $\mathcal{C}_{\zeta}$ with vertex at $\zeta$, isometric with $\mathcal{C}$ and satisfying $\mathcal{C}_{\zeta} \cap B\left(\zeta, r_{\zeta}\right) \subset D^{c}:=\mathbb{R}^{n} \backslash D$; see [6], [9], [10, [11] for more details. Then [10] Theorem 1.4] there exists a function $K_{\alpha}^{D}$, defined and continuous on $D \times D^{c}$ (the Poisson kernel for $D$ ), with the property that if $u$ is $\alpha$-harmonic in $D$ then

$$
u(x)=\int_{D^{c}} u(y) K_{\alpha}^{D}(x, y) d y .
$$

Thus every $\alpha$-harmonic function in $D$ is determined by its exterior values (its values on $D^{c}$ ). If $B$ is a Borel set in $D^{c}$, there exists a unique $\alpha$-harmonic function in $D$ with exterior values $\chi_{B}(y), y \in D^{c}$. This function is the $\alpha$-harmonic measure of $B$. It will be denoted by $\omega_{\alpha}(x, B, D), x \in \mathbb{R}^{n}$. For fixed $x \in D, \omega_{\alpha}(x, \cdot, D)$ is a probability measure supported on $D^{c}$; sometimes this measure will be denoted below by $\omega_{x}^{D}$.

Given a measure $\mu$ on $\mathbb{R}^{n}$, we let $U_{\alpha}^{\mu}$ be its Riesz potential,

$$
U_{\alpha}^{\mu}(x)=\mathcal{A}(n, \alpha) \int_{\mathbb{R}^{n}} \frac{d \mu(y)}{|x-y|^{n-\alpha}}, x \in \mathbb{R}^{n} .
$$

The Green function of an open set $D$ is

$$
G_{\alpha}^{D}(x, y)=U_{\alpha}^{\delta_{x}}(y)-U_{\alpha}^{\omega_{x}^{D}}(y), \quad x, y \in \mathbb{R}^{n} .
$$

The next theorem generalizes results for $\alpha=2$ proven in [25] and 3].

Theorem 3. Let $\alpha \in(0,2)$ and let $D$ be an open set in $\mathbb{R}^{n}, n \geq 2$. Then

(a) for $x, y \in D$,

$$
G_{\alpha}^{D}(x, y) \leq G_{\alpha}^{D^{*}}\left(x^{*}, y^{*}\right)
$$

(b) for $s \in D \cap \widehat{D}, y \in D$,

$$
G_{\alpha}^{D}(s, y)+G_{\alpha}^{D}(\hat{s}, y) \leq G_{\alpha}^{D^{*}}\left(s, y^{*}\right)+G_{\alpha}^{D^{*}}\left(\hat{s}, y^{*}\right) ;
$$

(c) for $s_{1}, s_{2} \in D \cap \widehat{D}$,

$$
\begin{aligned}
G_{\alpha}^{D}\left(s_{1}, s_{2}\right)+G_{\alpha}^{D}\left(\widehat{s_{1}}, s_{2}\right)+G_{\alpha}^{D}\left(\widehat{s_{1}}, \widehat{s_{2}}\right)+G_{\alpha}^{D}\left(\widehat{s_{1}}, \widehat{s_{2}}\right) \\
\quad \leq G_{\alpha}^{D^{*}}\left(s_{1}, s_{2}\right)+G_{\alpha}^{D^{*}}\left(\widehat{s_{1}}, s_{2}\right)+G_{\alpha}^{D^{*}}\left(\widehat{s_{1}}, \widehat{s_{2}}\right)+G_{\alpha}^{D^{*}}\left(\widehat{s_{1}}, \widehat{s_{2}}\right) .
\end{aligned}
$$

Assume in addition that $D$ is bounded with boundary satisfying an exterior cone condition. Let $B$ be a Borel set in $D^{c}$ such that $B^{*} \subset\left(D^{*}\right)^{c}$. Then

(d) for $x \in D$,

$$
\omega_{\alpha}(x, B, D) \leq \omega_{\alpha}\left(x^{*}, B^{*}, D^{*}\right)
$$

(e) for $s \in D \cap \widehat{D}$,

$$
\omega_{\alpha}(s, B, D)+\omega_{\alpha}(\hat{s}, B, D) \leq \omega_{\alpha}\left(s, B^{*}, D^{*}\right)+\omega_{\alpha}\left(\hat{s}, B^{*}, D^{*}\right) ;
$$

(f) for $x \in D, y \in D^{c}$,

$$
K_{\alpha}^{D}(x, y) \leq K_{\alpha}^{D^{*}}\left(x^{*}, y^{*}\right) ;
$$

(g) for $s \in D \cap \widehat{D}$ and $y \in D^{c}$ such that $y^{*} \in\left(D^{*}\right)^{c}$,

$$
K_{\alpha}^{D}(s, y)+K_{\alpha}^{D}(\hat{s}, y) \leq K_{\alpha}^{D^{*}}\left(s, y^{*}\right)+K_{\alpha}^{D^{*}}\left(\hat{s}, y^{*}\right) .
$$


We now turn to the probabilistic counterpart of the Riesz potential theory; see [4], 5], 6], [7], 9], [10], 11], 26]. The fractional Laplacian $\Delta^{\alpha / 2}$ is the characteristic operator of the symmetric $\alpha$-stable process $\left\{\mathrm{X}_{t}, t \in[0, \infty)\right\}$ in $\mathbb{R}^{n}$. This is a Lévy process (homogeneous and with independent increments) with transition density $p_{t}(x, y)=p_{t}(y, x)=p_{t}(x-y)$ (relative to the Lebesgue measure) uniquely determined by its Fourier transform

$$
\int_{\mathbb{R}^{n}} e^{i x \cdot \xi} p_{t}(x) d x=e^{-t|\xi|^{\alpha}} .
$$

When $\alpha=2$, we get a Brownian motion running at twice the speed. From now on we assume that $\alpha \in(0,2)$. Also, sometimes we will suppress the dependence of various quantities on $\alpha$; for example we will use the notation $\omega(x, B, D)$ or $G^{D}(x, y)$. The function $p_{t}(x)$ has the scaling property

$$
p_{t}(x)=\frac{1}{t^{n / \alpha}} p_{1}\left(\frac{x}{t^{1 / \alpha}}\right) .
$$

An explicit formula for $p_{1}(x)$ is given in [4]:

$$
p_{1}(x)=\frac{(2 \pi)^{n / 2}}{|x|^{n / 2-1}} \int_{0}^{\infty} e^{-r^{\alpha}} r^{n / 2} J_{(n-2) / 2}(|x| r) d r,
$$

where $J_{\nu}$ denotes the Bessel function of the first kind of order $\nu$. The probability measures and the corresponding expectations of the process $\left\{\mathrm{X}_{t}\right\}$ starting at $x \in \mathbb{R}^{n}$ will be denoted by $\mathbf{P}^{x}$ and $\mathbf{E}^{x}$. Thus, for the transition probabilities of the process we have

$$
P_{t}(x, A)=\mathbf{P}^{x}\left(\mathrm{X}_{t} \in A\right)=\int_{A} p_{t}(x-y) d y,
$$

where $A$ is a Borel subset of $\mathbb{R}^{n}$.

Let $D$ be a bounded open set in $\mathbb{R}^{n}$. We denote by $P_{t}^{D}(x, A), x \in D, A \subset D$, the transition probability of the process $\left\{\mathrm{X}_{t}\right\}$ killed on exiting $D$. The corresponding density will be denoted by $p_{t}^{D}(x, y)$. The next result describes the behavior of $P_{t}^{D}(x, A)$ under polarization. In the case of Brownian motion $(\alpha=2)$, it has been proved in [3] and [8].

Theorem 4. Let $D$ be an open set in $\mathbb{R}^{n}$ and $A$ be a Borel set in $D$. For $x \in D$, $s \in D \cap \widehat{D}$, and $t>0$ we have

$$
\begin{gathered}
P_{t}^{D}(x, A) \leq P_{t}^{D^{*}}\left(x^{*}, A^{*}\right) ; \\
P_{t}^{D}(s, A)+P_{t}^{D}(\hat{s}, A) \leq P_{t}^{D^{*}}\left(s, A^{*}\right)+P_{t}^{D^{*}}\left(\hat{s}, A^{*}\right) .
\end{gathered}
$$

We will see that Theorem 3 follows from Theorem 4 and various identities connecting the transition probabilities with harmonic measures, Green functions, and Poisson kernels. A review of the necessary probabilistic material will be presented in Section 2, which contains also some lemmas in preparation for the proof of Theorem 4 in Section 3. The proofs of Theorems 1, 2 and 3 are in Section 4. Finally, Section 5 contains some further results, including estimates for eigenvalues of Rayleigh-Faber-Krahn type and estimates related to a problem raised by Mattila [19]. The referee of the paper suggested a direct proof of Theorem 1 based on a Brascamp-Lieb-Luttinger-type inequality (cf. [2]). 


\section{Preliminaries}

The symmetric $\alpha$-stable process $\left\{\mathrm{x}_{t}\right\}$ is strong Markov with respect to the standard filtration $\left\{\mathcal{F}_{t}, t \geq 0\right\}$. The shift operator will be denoted by $\theta_{t}$. The potential kernel of $\left\{\mathrm{X}_{t}\right\}$ is the Riesz kernel:

$$
\int_{0}^{\infty} P_{t}(x, A) d t=\int_{A} k_{\alpha}(x-y) d y, \quad A \subset \mathbb{R}^{n}, \text { Borel. }
$$

It is well known that $\left\{\mathrm{X}_{t}\right\}$ is a strong Feller process and a Hunt process. For $A \subset \mathbb{R}^{n}$, we put

$$
T^{A}=\inf \left\{t>0: \mathrm{X}_{t} \notin A\right\},
$$

the first exit time from $A$. A Borel function $u$ defined on $\mathbb{R}^{n}$ is $\alpha$-harmonic in an open set $D \subset \mathbb{R}^{n}$ if and only if

$$
u(x)=\mathbf{E}^{x} u\left(\mathrm{X}_{T^{U}}\right), \quad x \in U,
$$

for every bounded open set $U$ with closure $\bar{U}$ contained in $D$. If $D \subset \mathbb{R}^{n}$ is open and $B$ is a Borel subset of $D^{c}$, then we have

$$
\omega_{\alpha}(x, B, D)=\mathbf{P}^{x}\left(\mathrm{x}_{T^{D}} \in B\right), x \in \mathbb{R}^{n} .
$$

The Green function of $D$ has also a probabilistic interpretation:

$$
G_{\alpha}^{D}(x, y)=\int_{0}^{\infty} p_{t}^{D}(x, y) d t, x, y \in D .
$$

The Green function and the Poisson kernel (when $D$ has the exterior cone property) are connected through the following formula (see, e.g., [10, Theorem 1.4]):

$$
K_{\alpha}^{D}(x, y)=\mathcal{A}(n, \alpha) \int_{D} \frac{G_{\alpha}^{D}(x, z)}{|z-y|^{n+\alpha}} d z, \quad x \in D, y \in D^{c} .
$$

When $D=B(0, r)$ (the ball of radius $r$ centered at the origin), the $\mathbf{P}^{x}$ distribution of $\mathrm{X}_{T^{D}}$ has the density function $P_{r}(x, \cdot)$ explicitly given by the formula [18, p. 122]

$$
P_{r}(x, y)=\Gamma\left(\frac{n}{2}\right) \frac{\sin \frac{\pi \alpha}{2}}{\pi^{n / 2+1}}\left[\frac{r^{2}-|x|^{2}}{|y|^{2}-r^{2}}\right]^{\alpha / 2} \frac{1}{|x-y|^{n}},|x|<r,|y|>r .
$$

The $\alpha$-capacity of a compact set $K \subset \mathbb{R}^{n}$ can also be characterized in terms of the symmetric stable process. We briefly describe this characterization, and refer the reader to 12], 13], 21], and [22 for more details. There is a unique measure $\pi_{K}$ having support contained in $K$ (the capacitary measure of $K$ ) such that

$$
\mathbf{P}^{x}\left(T^{K^{c}}<\infty\right)=\int_{K} k_{\alpha}(x-y) \pi_{K}(d y), x \in K^{c} .
$$

Then we have

$$
C_{\alpha}(K)=\pi_{K}(K) .
$$

[In the general setting of Hunt's probabilistic potential theory the identity (2.9) is the definition of capacity of $K$. It is a theorem of K.L.Chung and M.Rao ([12] Chapter 5], [13]) that, especially for the potential theory of certain kernels (that include the Riesz kernels), the probabilistic capacity $\pi_{K}(K)$ coincides with the capacity $C_{\alpha}(K)$ as defined in (1.4) in terms of energy.] 
The proof of Theorem 2 will be based on relations between hitting probabilities, Green functions, and $\alpha$-capacities established by S.Port [21], 22]. He proved the following identities:

$$
\begin{gathered}
C_{\alpha}(K)=\lim _{|x| \rightarrow \infty} \mathcal{A}(n, \alpha)^{-1}|x|^{n-\alpha} \mathbf{P}^{x}\left(T^{K^{c}}<\infty\right) ; \\
1-\mathbf{P}^{x}\left(T^{K^{c}}<\infty\right)=\lim _{|y| \rightarrow \infty} \mathcal{A}(n, \alpha)^{-1}|y|^{n-\alpha} G_{\alpha}^{K^{c}}(x, y), x \in K^{c} .
\end{gathered}
$$

We will now present some lemmas for later reference. From now on $\left\{\mathrm{X}_{t}, t \geq 0\right\}$ will denote a symmetric $\alpha$-stable process $(0<\alpha<2)$ with sample space $\Omega$. We may and do assume that $\left\{\mathrm{X}_{t}\right\}$ is the canonical version of the process [16, pp. 87-88]; so the elements $\omega$ of $\Omega$ are right-continuous functions defined on $[0, \infty)$ with values on $\mathbb{R}^{n}$ (paths).

Lemma 1. Let $S$ and $U$ be bounded open sets in $\mathbb{R}^{n}$ with boundaries satisfying an exterior cone condition. Suppose that $\bar{S} \cap \bar{U}=\emptyset$. Then:

(a) The function $s \mapsto \omega(s, U, S)$ is continuous on $\bar{S}$, and $\omega(s, U, S)=0$ for $s \in \partial S$.

(b) There is a positive constant $C$, depending only on $\alpha, S, U$, such that

$$
\max _{s \in \bar{S}} \omega(s, U, S) \leq C<1 .
$$

Proof. (a) It suffices to show that $\omega(s, U, S) \rightarrow 0$, when $S \ni s \rightarrow \partial S$. For $x \in \mathbb{R}^{n}$, let $\delta(x)=\operatorname{dist}(x, \partial S)$. By [9, Theorem 1.2],

$$
\begin{aligned}
0 & \leq \omega(s, U, S)=\int_{U} K^{S}(s, z) d z \\
& \leq \int_{U} \frac{c \delta(s)^{\alpha / 2}}{\delta(z)^{\alpha / 2}(1+\delta(z))^{\alpha / 2}|s-z|^{n}} d z \leq c \delta(s)^{\alpha / 2} m_{n}(U) .
\end{aligned}
$$

Since $\delta(s) \rightarrow 0$ as $s \rightarrow \partial S$, (a) is proved.

(b) Let $A$ be a fixed ball in $\mathbb{R}^{n} \backslash(\bar{S} \cup \bar{U})$. Let $s_{o}$ be a point in $S$ such that $\max _{s \in \bar{S}} \omega(s, U, S)=\omega\left(s_{o}, U, S\right)$. Then

$$
\omega\left(s_{o}, U, S\right) \leq 1-\omega\left(s_{o}, A, S\right):=C<1,
$$

and the lemma is proved.

Let $S, U$ be as in Lemma 1 and set $D=S \cup U$. For a symmetric $\alpha$-stable process starting from a point of $D$, we define the first entrance times

$$
\begin{aligned}
\tau_{1}^{S} & =\inf \left\{t>0: t \leq T^{D}, \mathbf{x}_{t} \in S\right\}, \\
\tau_{1}^{U} & =\inf \left\{t>0: t \leq T^{D}, \mathbf{x}_{t} \in U\right\},
\end{aligned}
$$

and the first exit times

$$
\begin{aligned}
& T_{1}^{S}=\inf \left\{t>\tau_{1}^{S}: t \leq T^{D}, \mathbf{x}_{t} \notin S\right\}, \\
& T_{1}^{U}=\inf \left\{t>\tau_{1}^{U}: t \leq T^{D}, \mathbf{x}_{t} \notin U\right\} .
\end{aligned}
$$


We also define inductively four sequences $\left\{\tau_{k}^{S}\right\},\left\{\tau_{k}^{U}\right\},\left\{T_{k}^{S}\right\},\left\{T_{k}^{U}\right\}, k \geq 1$, of Markov times as follows: For $k \geq 2$, we set

$$
\begin{gathered}
\tau_{k}^{S}=\inf \left\{t>T_{k-1}^{S}: t \leq T^{D}, \mathrm{x}_{t} \in S\right\} ; \\
\tau_{k}^{U}=\inf \left\{t>T_{k-1}^{U}: t \leq T^{D}, \mathrm{x}_{t} \in U\right\} ; \\
T_{k}^{S}=\inf \left\{t>\tau_{k}^{S}: t \leq T^{D}, \mathrm{x}_{t} \notin S\right\} ; \\
T_{k}^{U}=\inf \left\{t>\tau_{k}^{U}: t \leq T^{D}, \mathrm{x}_{t} \notin U\right\} .
\end{gathered}
$$

As usual, if the set $\left\{t>\tau_{k}^{S}: t \leq T^{D}, \mathrm{x}_{t} \notin S\right\}$ is empty, we set $T_{k}^{S}=\infty$, and similarly for the other Markov times. Thus, for example, $T_{k}^{U}(\omega)$ is the moment when the path $\omega$ makes the $k$ th jump from $U$ to $S$ (having stayed inside $D$ until that moment). For $t>0$, we will say that a path $\omega$ visits $U$ an infinite number of times in the time interval $(0, t]$ if $T_{k}^{U} \leq t$ for all $k \geq 1$. Otherwise, we will say that $\omega$ visits $U$ in the time interval $(0, t]$ a finite number of times. Note that if the process starts from a point in $S$, then almost surely $T_{1}^{S}<T_{1}^{U}$, because $U$ is open and the process almost surely spends a positive amount of time in every ball. The inequality $T_{1}^{S}<T_{1}^{U}$ implies $0<T_{1}^{S}<T_{1}^{U}<T_{2}^{S}<T_{2}^{U}<\ldots$ provided that these exit times are finite.

Lemma 2. With the above assumptions, almost every path starting from $s_{o} \in S$ visits $U$ a finite number of times in the time interval $(0, t]$.

Proof. By Lemma 1, $\omega(s, U, S) \leq C<1$, for all $s \in S$. So

$$
\mathbf{P}^{s_{o}}\left(T_{1}^{U} \leq t\right) \leq \mathbf{P}^{s_{o}}\left(\mathrm{x}_{T_{1}^{S}} \in U\right)=\omega\left(s_{o}, U, S\right) \leq C .
$$

By the strong Markov property, $\mathbf{P}^{s_{o}}\left(T_{k}^{S} \leq t\right) \leq C^{k}$. Hence the probability that $\omega$ visits $U$ an infinite number of times in the time interval $(0, t]$ is equal to

$$
\mathbf{P}^{s_{o}}\left(\bigcap_{k=1}^{\infty}\left\{T_{k}^{U} \leq t\right\}\right)=\lim _{k \rightarrow \infty} \mathbf{P}^{s_{o}}\left(T_{k}^{U} \leq t\right) \leq \lim _{k \rightarrow \infty} C^{k}=0 .
$$

Lemma 3. Suppose $x, y \in \mathbb{R}^{n}$ are such that $|x-y| \geq \delta>0$. Then there is a constant $C=C(\delta, \alpha)$ such that $p_{t}(x, y) \leq C$, for all $t>0$.

Proof. The lemma follows from (1.23), (1.24), and the boundedness of Bessel functions of the first kind.

Lemma 4. Let $\left\{D_{j}\right\}$ be an increasing sequence of open sets and $D=\bigcup_{j=1}^{\infty} D_{j}$. Then

$$
\lim _{j \rightarrow \infty} p_{t}^{D_{j}}(x, y)=p_{t}^{D}(x, y),
$$

for every $t>0$ and all $x, y$ in $D$.

Proof. The proof is the same as the corresponding result for Brownian motion presented in [23, pp. 39-40]. (In this proof we need the estimate of Lemma B)

Lemma 5. Let $S$ be a bounded open set in $\mathbb{R}^{n}$ with boundary satisfying an exterior cone condition. Assume that $S$ is symmetric with respect to $\Pi_{n-1}$. Then:

(a) If $s \in S \cap \Pi_{n-1}$ and $y \in S_{+}$, then

$$
p_{t}^{S}(s, y)=p_{t}^{S}(s, \hat{y}), t>0 .
$$


(b) If $x, y \in S$, and $t>0$, then

$$
\begin{aligned}
p_{t}^{S}(x, y) & =p_{t}^{S}(\hat{x}, \hat{y}), \\
p_{t}^{S}(x, y)+p_{t}^{S}(\hat{x}, y) & =p_{t}^{S}(x, \hat{y})+p_{t}^{S}(\hat{x}, \hat{y}) .
\end{aligned}
$$

(c) If $s, y \in S_{+}$and $t>0$, then

$$
p_{t}^{S}(s, y) \geq p_{t}^{S}(s, \hat{y}) .
$$

Proof. The equalities in (a) and (b) follow from the reflection invariance of the symmetric stable process (see [16, $\S 10.27$, Theorem 10.14]). To prove (c) we fix $a>0$ and $y \in S_{+}$, and define

$$
f(t, s)=p_{t}^{S}(s, y)-p_{t}^{S}(s, \hat{y}), \quad t \in(0, a], s \in S_{+} .
$$

By (a) and the regularity of $\partial\left(S_{+}\right), f$ is continuous on $(0, a] \times \overline{S_{+}}$, and $f(t, s) \rightarrow 0$ as $s \rightarrow \partial\left(S_{+}\right)$, for all $t \in(0, a]$. Also $f(t, s) \rightarrow \delta_{s y}-\delta_{s \hat{y}} \geq 0$, as $t \rightarrow 0$, for all $s \in S_{+}$. Suppose now that $f$ has a strictly negative minimum on $(0, a] \times \overline{S_{+}}$attained at the point $\left(t_{o}, s_{o}\right)$. By the convergence properties we showed above, $s_{o} \in S_{+}$. For the fractional Laplacian applied to the function $s \mapsto f\left(t_{o}, s\right)$, we have

$$
\Delta^{\alpha / 2} f\left(t_{o}, s_{o}\right)=\mathcal{A}(n, \alpha) \int_{\mathbb{R}^{n}} \frac{f\left(t_{o}, s\right)-f\left(t_{o}, s_{o}\right)}{\left|s-s_{o}\right|^{n+\alpha}} d s>0 .
$$

The inequality is strict because $f\left(t_{o}, s\right)=0$ for $s \notin S, f$ is continuous, and $f\left(t_{o}, s_{o}\right)<0$. On the other hand, since $\left(t_{o}, s_{o}\right)$ is a minimum point, $\frac{\partial f}{\partial t}\left(t_{o}, s_{o}\right) \leq 0$ (in fact, this derivative is 0 unless $t_{o}=a$ ). But since $\Delta^{\alpha / 2}$ is the infinitesimal generator of the semigroup associated to the symmetric stable process, we have (see [16, p. 55])

$$
0<\Delta^{\alpha / 2} f\left(t_{o}, s_{o}\right)=\frac{\partial f}{\partial t}\left(t_{o}, s_{o}\right) \leq 0 .
$$

This contradiction shows that $f(t, s) \geq 0$, for $t \in(0, a]$ and $s \in S_{+}$. Since $a$ is arbitrary, this inequality holds for all $t>0$.

Lemma 6. Let $S$ be as in Lemma可, and let $B$ be a Borel set in $\mathbb{R}_{+}^{n} \backslash S$. Then for $x \in S_{+}$,

$$
\begin{aligned}
\omega(x, B, S) & =\omega(\hat{x}, \widehat{B}, S) ; \\
\omega(x, \widehat{B}, S) & =\omega(\hat{x}, B, S) ; \\
\omega(x, B, S) & \geq \omega(\hat{x}, B, S) ; \\
\omega(x, B, S) & \geq \omega(x, \widehat{B}, S) ; \\
\omega(x, B, S)+\omega(\hat{x}, B, S) & =\omega(x, \widehat{B}, S)+\omega(\hat{x}, \widehat{B}, S) .
\end{aligned}
$$

Proof. By Lemma 5, for all $t>0, x \in S, y \in S$, we have $p_{t}^{S}(x, y)=p_{t}^{S}(\hat{x}, \hat{y})$. Hence $G^{S}(x, y)=G^{S}(\hat{x}, \hat{y})$. Because of (2.6), this implies $K^{S}(x, y)=K^{S}(\hat{x}, \hat{y})$ for $x \in S$ and $z \in S^{c}$. Now using (1.12) we obtain (2.24). The other assertions of the lemma are proved similarly. 
Lemma 7. Let $U$ be a bounded open set in $\mathbb{R}_{+}^{n}$ with boundary satisfying an exterior cone condition. Let $B$ be a Borel set in $\mathbb{R}_{+}^{n}$. Then for $u \in U$,

$$
\begin{aligned}
\omega(u, B, U) & =\omega(\hat{u}, \widehat{B}, \widehat{U}) ; \\
\omega(u, \widehat{B}, U) & =\omega(\hat{u}, B, \widehat{U}) ; \\
\omega(u, B, U) & \geq \omega(\hat{u}, B, \widehat{U}) ; \\
\omega(u, B, U) & \geq \omega(u, \widehat{B}, U) .
\end{aligned}
$$

Proof. If $U$ is a ball then (2.29)-(2.32) follow easily from the explicit expression for the Poisson kernel (2.7). The general case follows by applying the strong Markov property, as was done in [6. Proof of Lemma 6].

Lemma 8. Let $U$ be a bounded open set in $\mathbb{R}_{+}^{n}$ with boundary satisfying an exterior cone condition. Let $S_{1}$ be a Borel set in $\mathbb{R}_{+}^{n}$ such that $\overline{S_{1}} \cap \bar{U}=\emptyset$. Let $T$ be a Borel subset of $(0, \infty)$. Then for $u \in U$,

$$
\mathbf{P}^{u}\left(\mathrm{X}_{T_{1}^{U}} \in S_{1}, T_{1}^{U} \in T\right) \geq \mathbf{P}^{u}\left(\mathrm{X}_{T_{1}^{U}} \in \widehat{S_{1}}, T_{1}^{U} \in T\right) .
$$

Proof. It suffices to prove the inequality when $T$ is an open interval. For $0 \leq t_{1}<$ $t_{2}<+\infty$, we have by the Markov property

$$
\begin{aligned}
& \mathbf{P}^{u}\left(\mathrm{x}_{T_{1}^{U}} \in S_{1}, t_{1}<T_{1}^{U}<t_{2}\right) \\
= & \int_{U} \mathbf{P}^{u}\left(\mathrm{x}_{t_{1}} \in d u_{1}, t_{1}<T_{1}^{U}\right) \mathbf{P}^{u_{1}}\left(\mathrm{x}_{T_{1}^{U}} \in S_{1}, T_{1}^{U}<t_{2}-t_{1}\right) \\
= & \int_{U} P_{t_{1}}^{U}\left(u, d u_{1}\right)\left[\mathbf{P}^{u_{1}}\left(\mathrm{x}_{T_{1}^{U}} \in S_{1}\right)-\mathbf{P}^{u_{1}}\left(\mathrm{x}_{T_{1}^{U}} \in S_{1}, T_{1}^{U}>t_{2}-t_{1}\right)\right] \\
= & \int_{U} P_{t_{1}}^{U}\left(u, d u_{1}\right)\left[\omega\left(u_{1}, S_{1}, U\right)-\int_{U} P_{t_{2}-t_{1}}^{U}\left(u_{1}, d u_{2}\right) \omega\left(u_{2}, S_{1}, U\right)\right] .
\end{aligned}
$$

Similarly we have

$$
\begin{aligned}
& \mathbf{P}^{u}\left(\mathrm{X}_{T_{1}^{U}} \in \widehat{S_{1}}, t_{1}<T_{1}^{U}<t_{2}\right) \\
= & \int_{U} P_{t_{1}}^{U}\left(u, d u_{1}\right)\left[\omega\left(u_{1}, \widehat{S_{1}}, U\right)-\int_{U} P_{t_{2}-t_{1}}^{U}\left(u_{1}, d u_{2}\right) \omega\left(u_{2}, \widehat{S_{1}}, U\right)\right] .
\end{aligned}
$$

Therefore, to prove (2.33), it suffices to prove that for all $u_{1} \in U$,

$$
\begin{aligned}
& \int_{U} P_{t_{2}-t_{1}}^{U}\left(u_{1}, d u_{2}\right) {\left[\omega\left(u_{2}, S_{1}, U\right)-\omega\left(u_{2}, \widehat{S_{1}}, U\right)\right] } \\
& \leq \omega\left(u_{1}, S_{1}, U\right)-\omega\left(u_{1}, \widehat{S_{1}}, U\right) .
\end{aligned}
$$

By Lemma 7 the function $u_{1} \mapsto \omega\left(u_{1}, S_{1}, U\right)-\omega\left(u_{1}, \widehat{S_{1}}, U\right)$ is nonnegative in $U$. It is also $\alpha$-harmonic in $U$, and therefore it has the averaging property (2.3). Hence (see 17, Chapter XII, Theorem 12.4]) it is an excessive function for the symmetric stable process killed on exiting $U$. Now (2.34) follows from the definition of excessive functions.

Lemma 9. Let $S, U$ be open sets in $\mathbb{R}^{n}$ with $\bar{S} \cap \bar{U}=\emptyset$. Let $A$ be a Borel subset of $S, s \in S$, and $t>0$. For the event

$$
\mathcal{E}=\left\{\omega \in \Omega: \mathrm{X}_{T_{1}^{S}} \in U, \mathrm{X}_{T_{1}^{U}} \in S, \mathrm{X}_{t} \in A, T_{1}^{S}<t<T_{2}^{S}\right\},
$$


we have

$$
\begin{array}{r}
\mathbf{P}^{s}(\mathcal{E})=\int_{0}^{t} \int_{U} \mathbf{P}^{s}\left(\omega_{o}: \mathrm{X}_{T_{1}^{S}}\left(\omega_{o}\right) \in d u, T_{1}^{S}\left(\omega_{o}\right) \in d t_{1}\right) \\
\times \mathbf{P}^{u}\left(\omega_{1}: \mathrm{X}_{T_{1}^{U}}\left(\omega_{1}\right) \in S, \mathrm{x}_{t-t_{1}}\left(\omega_{1}\right) \in A, t-t_{1}<T_{1}^{S}\left(\omega_{1}\right)\right) .
\end{array}
$$

Proof. We consider the function $F:[0, \infty) \times \Omega \rightarrow \mathbb{R}$ defined by

$$
F\left(t_{1}, \omega\right)=\left\{\begin{array}{l}
1, \text { if } 0<t-t_{1}<T_{1}^{S}(\omega), \mathrm{x}_{T_{1}^{U}}(\omega) \in S, \mathrm{x}_{t-t_{1}}(\omega) \in A \\
0, \text { otherwise. }
\end{array}\right.
$$

We also let

$$
\mathcal{E}_{o}=\left\{\omega_{o}: \mathrm{X}_{T_{1}^{S}}\left(\omega_{o}\right) \in U, T_{1}^{S}\left(\omega_{o}\right)<t\right\},
$$

and denote by $\mathrm{I}_{\mathcal{E}_{o}}$ the indicator (characteristic) function of $\mathcal{E}_{o}$. For $\omega_{o} \in \mathcal{E}_{o}$ we consider the event

$$
\mathcal{E}_{1}\left(\omega_{o}\right)=\left\{\omega_{1}: 0<t-T_{1}^{S}\left(\omega_{o}\right)<T_{1}^{S}\left(\omega_{1}\right), \mathrm{X}_{T_{1}^{U}} \in S, \mathrm{X}_{t-T_{1}^{S}\left(\omega_{o}\right)}\left(\omega_{1}\right) \in A\right\} .
$$

Then we have

$$
\begin{aligned}
\mathbf{P}^{s}(\mathcal{E})= & \mathbf{E}^{s}\left[\mathrm{I}_{\mathcal{E}_{o}} F\left(T_{1}^{S}, \theta_{T_{1}^{S}}(\omega)\right)\right] \\
= & \mathbf{E}^{s}\left[\mathrm{I}_{\mathcal{E}_{o}} \mathbf{E}^{s}\left[F\left(T_{1}^{S}, \theta_{T_{1}^{S}}(\omega)\right) \mid \mathcal{F}_{T_{1}^{S}}\right]\right] \\
= & \mathbf{E}^{s}\left[\left.\mathrm{I}_{\mathcal{E}_{o}} \mathbf{E}^{\mathrm{x}_{T_{1}^{S}}} F\left(t_{1}, \omega\right)\right|_{\left.t_{1}=T_{T_{1}^{S}}\right]}\right] \\
= & \mathbf{E}^{s}\left[\mathrm{I}_{\mathcal{E}_{o}}\left(\omega_{o}\right) \mathbf{P}^{\mathrm{x}_{T_{1}^{S}}\left(\omega_{o}\right)}\left(\mathcal{E}_{1}\left(\omega_{o}\right)\right]\right. \\
= & \int_{\mathcal{E}_{o}} \mathbf{P}^{s}\left(d \omega_{o}\right) \mathbf{P}^{\mathrm{x}_{T_{1}^{S}\left(\omega_{o}\right)}}\left(\mathcal{E}_{1}\left(\omega_{o}\right)\right) \\
= & \int_{0}^{t} \int_{U} \mathbf{P}^{s}\left(\omega_{o}: \mathrm{X}_{T_{1}^{S}}\left(\omega_{o}\right) \in d u, T_{1}^{S}\left(\omega_{o}\right) \in d t_{1}\right) \\
& \times \mathbf{P}^{u}\left(\omega_{1}: \mathrm{X}_{T_{1}^{U}}\left(\omega_{1}\right) \in S, \mathrm{x}_{t-t_{1}}\left(\omega_{1}\right) \in A, t-t_{1}<T_{1}^{S}\left(\omega_{1}\right)\right)
\end{aligned}
$$

In the first equality we used the facts

$$
\begin{gathered}
\theta_{T_{1}^{S}}\left\{\mathrm{X}_{t-T_{1}^{S}} \in A\right\}=\left\{\mathrm{x}_{t} \in A\right\} \\
\left\{t-T_{1}^{S}<\theta_{T_{1}^{S}} T_{1}^{S}\right\}=\left\{t<T_{1}^{S}+\theta_{T_{1}^{S}} T_{1}^{S}\right\}=\left\{t<T_{2}^{S}\right\},
\end{gathered}
$$

for paths $\omega \in \mathcal{E}_{o}$ starting from $s$. The third equality comes from the strong Markov property. In the last equality we used a change of variables.

\section{Proof of Theorem 4}

We will prove (1.27). The proof of (1.26) is similar. Let $D, A$ be as in the statement of Theorem 4 and let $t>0$. Let $D=S \cup U \cup V$ be the decomposition of $D$ described in the introduction, i.e. $S=D \cap \widehat{D}, U=D_{+} \backslash S$, and $V=D_{-} \backslash S$. We will first prove (1.27) under the following additional assumptions:

Assumption A: The Borel set $A$ is bounded.

Assumption B: The open set $D$ is the union of a finite number of (open) cubes. Assumption C: $\bar{S} \cap \bar{U}=\emptyset, \bar{S} \cap \bar{V}=\emptyset, \bar{U} \cap \bar{V}=\emptyset$. 
Note that under these assumptions the sets $S, U, V$ are open, bounded, and their boundaries satisfy an exterior cone condition. We will later remove Assumptions A, B, C.

We decompose $A$ into three disjoint sets: $A=A_{1} \cup A_{2} \cup A_{3}:=(A \cap S) \cup(A \cap U) \cup$ $(A \cap V)$. To prove (1.27), it suffices to prove it with $A_{i}$ in place of $A(i=1,2,3)$. We will prove it for $A_{1}$; the proof for $A_{2}$ and $A_{3}$ is similar. So from now on we assume that $A \subset S$.

For every $s \in S$, we consider the sets of paths

$$
\begin{aligned}
& \mathcal{A}_{s}=\left\{\omega \in \Omega: \mathrm{X}_{0}=s, \mathrm{X}_{t} \in A, t<T^{D}\right\}, \\
& \mathcal{A}_{s}^{*}=\left\{\omega \in \Omega: \mathrm{X}_{0}=s, \mathrm{X}_{t} \in A^{*}, t<T^{D^{*}}\right\} .
\end{aligned}
$$

Then $\mathbf{P}\left(\mathcal{A}_{s}\right)=P_{t}^{D}(s, A)$ and $\mathbf{P}\left(\mathcal{A}_{s}^{*}\right)=P_{t}^{D^{*}}\left(s, A^{*}\right)$. Therefore, in order to prove (1.27), it suffices to prove that

$$
\mathbf{P}\left(\mathcal{A}_{s}\right)+\mathbf{P}\left(\mathcal{A}_{\hat{s}}\right) \leq \mathbf{P}\left(\mathcal{A}_{s}^{*}\right)+\mathbf{P}\left(\mathcal{A}_{\hat{s}}^{*}\right) .
$$

By Lemma 2, each path $\omega \in \mathcal{A}_{s}$ visits $S, U$, and $V$ a finite number of times. Because of this fact we can decompose $\mathcal{A}_{s}$ into an infinite number of disjoint sets as follows:

$$
\begin{aligned}
\mathcal{A}_{s}= & {[S] \cup[S U S] \cup[S V S] \cup[S U V S] \cup[S V U S] \cup[S U S U S] } \\
& \cup[S U S V S] \cup[S V S U S] \cup[S V S V S] \cup[S U V U S] \cup \ldots
\end{aligned}
$$

We call the above sets the bracket sets of $\mathcal{A}_{s}$. In order to give the reader the idea of their definition we describe the first four such bracket sets precisely:

$$
\begin{aligned}
{[S] } & =\left\{\omega \in \mathcal{A}_{s}: t<T_{1}^{S}\right\} \\
{[S U S] } & =\left\{\omega \in \mathcal{A}_{s}: \mathrm{X}_{T_{1}^{S}} \in U, \mathrm{X}_{T_{1}^{U}} \in S, T_{1}^{S}<t<T_{2}^{S}\right\} ; \\
{[S V S] } & =\left\{\omega \in \mathcal{A}_{s}: \mathrm{X}_{T_{1}^{S}} \in V, \mathrm{X}_{T_{1}^{V}} \in S, T_{1}^{S}<t<T_{2}^{S}\right\} ; \\
{[S U V S] } & =\left\{\omega \in \mathcal{A}_{s}: \mathrm{X}_{T_{1}^{S}} \in U, \mathrm{X}_{T_{1}^{U}} \in V, \mathrm{X}_{T_{1}^{V}} \in S, T_{1}^{S}<t<T_{2}^{S}\right\} .
\end{aligned}
$$

A general bracket set of $\mathcal{A}_{s}$ is denoted by a "word" in brackets; the first letter of the word is $S$, the last letter of the word is $S$, and the letters in between are $S, U$, or $V$; each letter is followed by a different letter. The rigorous definition should be clear from the definition of the first four bracket sets above. Intuitively, the letters in brackets describe the history of the corresponding paths from their starting point $s \in S$ until the moment $t$ at which the paths are in $A \subset S$. For example [SUVSUS] is the set of paths that start from $s$ and jump successively from $S$ to $U$, then to $V$, then to $S$, then to $U$, then to $S$, and finally, before exiting $S$ again, at time $t$ they are in $A$.

The set $\mathcal{A}_{s}^{*}$ is also decomposed into bracket sets which are similar to those of $\mathcal{A}_{s}$; the only notational difference is that we now have $\widehat{V}$ in place of $V$. The bracket sets of $\mathcal{A}_{s}^{*}$ will have the upper index $*$ to distinguish them from the bracket sets of $\mathcal{A}_{s}$ :

$$
\begin{aligned}
\mathcal{A}_{s}^{*}= & {[S]^{*} \cup[S U S]^{*} \cup[S \widehat{V} S]^{*} \cup[S U \widehat{V} S]^{*} \cup[S \widehat{V} U S]^{*} \cup[S U S U S]^{*} } \\
& \cup[S U S \widehat{V} S]^{*} \cup[S \widehat{V} S U S]^{*} \cup[S \widehat{V} S \widehat{V} S]^{*} \cup[S U \widehat{V} U S]^{*} \cup \ldots
\end{aligned}
$$

We now compare the probability of the bracket sets of $\mathcal{A}_{s}$ with the probability of the correponding bracket sets of $\mathcal{A}_{s}^{*}$. For example, we show that

$$
\mathbf{P}^{s}([S V S])+\mathbf{P}^{\hat{s}}([S V S]) \leq \mathbf{P}^{s}\left([S \widehat{V} S]^{*}\right)+\mathbf{P}^{\hat{s}}\left([S \widehat{V} S]^{*}\right) .
$$


By using Lemma 9 iteratively we obtain

$$
\begin{aligned}
& \mathbf{P}^{s}([S V S])=\int_{0}^{t} \int_{V} \mathbf{P}^{s}\left(\mathrm{x}_{T_{1}^{S}} \in d v, T_{1}^{S} \in d t_{1}\right) \\
& \quad \times \int_{0}^{t-t_{1}} \int_{S} \mathbf{P}^{v}\left(\mathrm{X}_{T_{1}^{V}} \in d s_{1}, T_{1}^{V} \in d t_{2}\right) \mathbf{P}^{s_{1}}\left(\mathrm{X}_{t-t_{1}-t_{2}} \in A, t-t_{1}-t_{2}<T^{S}\right) .
\end{aligned}
$$

For typographical convenience we set

$$
\begin{aligned}
\left\{\mathbf{P}^{s}\right\} & :=\mathbf{P}^{s}\left(\mathrm{x}_{T_{1}^{S}} \in d v, T_{1}^{S} \in d t_{1}\right), \\
\left\{\mathbf{P}^{v}\right\} & :=\mathbf{P}^{v}\left(\mathrm{x}_{T_{1}^{V}} \in d s_{1}, T_{1}^{V} \in d t_{2}\right), \\
\left\{\mathbf{P}^{s_{1}}\right\} & :=\mathbf{P}^{s_{1}}\left(\mathrm{x}_{t-t_{1}-t_{2}} \in A, t-t_{1}-t_{2}<T^{S}\right) .
\end{aligned}
$$

With this notation (3.3) becomes

$$
\mathbf{P}^{s}([S V S])=\int_{0}^{t} \int_{V}\left\{\mathbf{P}^{s}\right\} \int_{0}^{t-t_{1}} \int_{S}\left\{\mathbf{P}^{v}\right\}\left\{\mathbf{P}^{s_{1}}\right\} .
$$

We define a new measure $\widehat{\mathbf{P}}^{v}$ by

$$
\widehat{\mathbf{P}}^{v}\left(\mathrm{X}_{T_{1}^{V}} \in \Sigma_{1}, T_{1}^{V} \in T\right)=\mathbf{P}^{v}\left(\mathrm{x}_{T_{1}^{V}} \in \widehat{\Sigma_{1}}, T_{1}^{V} \in T\right), \Sigma \subset S, T \subset(0, \infty) .
$$

We will also use the abbreviations

$$
\begin{aligned}
\left\{\widehat{\mathbf{P}}^{v}\right\} & :=\widehat{\mathbf{P}}^{v}\left(\mathrm{X}_{T_{1}^{V}} \in d s_{1}, T_{1}^{V} \in d t_{2}\right), \\
\left\{\mathbf{P}^{\widehat{s_{1}}}\right\} & :=\mathbf{P}^{\widehat{s_{1}}}\left(\mathrm{X}_{t-t_{1}-t_{2}} \in A, t-t_{1}-t_{2}<T^{S}\right), \\
\left\{\mathbf{P}^{\hat{s}}\right\} & :=\mathbf{P}^{\hat{s}}\left(\mathrm{X}_{T_{1}^{S}} \in d v, T_{1}^{S} \in d t_{1}\right),
\end{aligned}
$$

and write (3.4) as

$$
\begin{aligned}
\mathbf{P}^{s}([S V S]= & \int_{0}^{t} \int_{V}\left\{\mathbf{P}^{s}\right\} \int_{0}^{t-t_{1}} \int_{S_{+}}\left\{\mathbf{P}^{v}\right\}\left\{\mathbf{P}^{s_{1}}\right\} \\
& +\int_{0}^{t} \int_{V}\left\{\mathbf{P}^{s}\right\} \int_{0}^{t-t_{1}} \int_{S_{+}}\left\{\widehat{\mathbf{P}}^{v}\right\}\left\{\mathbf{P}^{\widehat{s_{1}}}\right\} .
\end{aligned}
$$

By adding and subtracting the same term, we obtain

$$
\begin{aligned}
\mathbf{P}^{s}([S V S])= & \int_{0}^{t} \int_{V}\left\{\mathbf{P}^{s}\right\} \int_{0}^{t-t_{1}} \int_{S_{+}}\left(\left\{\widehat{\mathbf{P}}^{v}\right\}-\left\{\mathbf{P}^{v}\right\}\right)\left\{\mathbf{P}^{\widehat{s_{1}}}\right\} \\
& +\int_{0}^{t} \int_{V}\left\{\mathbf{P}^{s}\right\} \int_{0}^{t-t_{1}} \int_{S_{+}}\left\{\mathbf{P}^{v}\right\}\left(\left\{\mathbf{P}^{s_{1}}\right\}+\left\{\mathbf{P}^{\widehat{s_{1}}}\right\}\right) .
\end{aligned}
$$

The analogous formula for $\mathbf{P}^{\hat{s}}[S V S]$ is

$$
\begin{aligned}
\mathbf{P}^{\hat{s}}([S V S])= & \int_{0}^{t} \int_{V}\left\{\mathbf{P}^{\hat{s}}\right\} \int_{0}^{t-t_{1}} \int_{S_{+}}\left(\left\{\widehat{\mathbf{P}}^{v}\right\}-\left\{\mathbf{P}^{v}\right\}\right)\left\{\mathbf{P}^{\widehat{s_{1}}}\right\} \\
& +\int_{0}^{t} \int_{V}\left\{\mathbf{P}^{\hat{s}}\right\} \int_{0}^{t-t_{1}} \int_{S_{+}}\left\{\mathbf{P}^{v}\right\}\left(\left\{\mathbf{P}^{s_{1}}\right\}+\left\{\mathbf{P}^{\widehat{s_{1}}}\right\}\right) .
\end{aligned}
$$


By (3.6) and (3.7),

$$
\begin{aligned}
& \mathbf{P}^{s}([S V S])+\mathbf{P}^{\hat{s}}([S V S]) \\
& =\int_{0}^{t} \int_{V}\left(\left\{\mathbf{P}^{s}\right\}+\left\{\mathbf{P}^{\hat{s}}\right\}\right) \int_{0}^{t-t_{1}} \int_{S_{+}}\left(\left\{\widehat{\mathbf{P}}^{v}\right\}-\left\{\mathbf{P}^{v}\right\}\right)\left\{\mathbf{P}^{\widehat{s_{1}}}\right\} \\
& +\int_{0}^{t} \int_{V}\left(\left\{\mathbf{P}^{s}\right\}+\left\{\mathbf{P}^{\hat{s}}\right\}\right) \int_{0}^{t-t_{1}} \int_{S_{+}}\left\{\mathbf{P}^{v}\right\}\left(\left\{\mathbf{P}^{s_{1}}\right\}+\left\{\mathbf{P}^{\widehat{s_{1}}}\right\}\right) .
\end{aligned}
$$

Similarly,

$$
\begin{aligned}
& \mathbf{P}^{s}\left([S \widehat{V} S]^{*}\right)+\mathbf{P}^{\hat{s}}\left([S \widehat{V} S]^{*}\right) \\
& =\int_{0}^{t} \int_{V}\left(\left\{\widehat{\mathbf{P}}^{s}\right\}+\left\{\widehat{\mathbf{P}}^{\hat{s}}\right\}\right) \int_{0}^{t-t_{1}} \int_{S_{+}}\left(\left\{\mathbf{P}^{\hat{v}}\right\}-\left\{\widehat{\mathbf{P}}^{\hat{v}}\right\}\right)\left\{\mathbf{P}_{*}^{s_{1}}\right\} \\
& +\int_{0}^{t} \int_{V}\left(\left\{\widehat{\mathbf{P}}^{s}\right\}+\left\{\widehat{\mathbf{P}}^{\hat{s}}\right\}\right) \int_{0}^{t-t_{1}} \int_{S_{+}}\left\{\widehat{\mathbf{P}}^{\hat{v}}\right\}\left(\left\{\mathbf{P}_{*}^{s_{1}}\right\}+\left\{\mathbf{P}_{*}^{\widehat{s_{1}}}\right\}\right),
\end{aligned}
$$

where we used the abbreviations

$$
\begin{aligned}
\left\{\widehat{\mathbf{P}}^{s}\right\} & :=\mathbf{P}^{s}\left(\mathrm{x}_{T_{1}^{S}} \in \widehat{d v}, T_{1}^{S} \in d t_{1}\right), \\
\left\{\widehat{\mathbf{P}}^{\hat{s}}\right\} & :=\mathbf{P}^{\hat{s}}\left(\mathrm{x}_{T_{1}^{S}} \in \widehat{d v}, T_{1}^{S} \in d t_{1}\right), \\
\left\{\mathbf{P}^{\hat{v}}\right\} & :=\mathbf{P}^{\hat{v}}\left(\mathrm{X}_{T_{1}^{\widehat{V}}} \in d s_{1}, T_{1}^{\widehat{V}} \in d t_{2}\right), \\
\left\{\widehat{\mathbf{P}}^{\hat{v}}\right\} & :=\mathbf{P}^{\hat{v}}\left(\mathrm{X}_{T_{1}^{\widehat{V}}} \in \widehat{d s_{1}}, T_{1}^{\widehat{V}} \in d t_{2}\right), \\
\left\{\mathbf{P}_{*}^{s_{1}}\right\} & :=\mathbf{P}^{s_{1}}\left(\mathrm{X}_{t-t_{1}-t_{2}} \in A^{*}, t-t_{1}-t_{2}<T^{S}\right), \\
\left\{\mathbf{P}_{*}^{\widehat{s_{1}}}\right\} & :=\mathbf{P}^{\widehat{s_{1}}}\left(\mathrm{x}_{t-t_{1}-t_{2}} \in A^{*}, t-t_{1}-t_{2}<T^{S}\right) .
\end{aligned}
$$

By (3.8) and (3.9), in order to prove (3.2), it suffices to prove the following claims:

Claim 1: For $s \in S, V_{1} \subset V, T \subset(0, \infty)$,

$$
\begin{aligned}
& \mathbf{P}^{s}\left(\mathrm{x}_{T_{1}^{S}} \in V_{1}, T_{1}^{S} \in T\right)+\mathbf{P}^{\hat{s}}\left(\mathrm{x}_{T_{1}^{S}} \in V_{1}, T_{1}^{S} \in T\right) \\
& =\mathbf{P}^{s}\left(\mathrm{x}_{T_{1}^{S}} \in \widehat{V_{1}}, T_{1}^{S} \in T\right)+\mathbf{P}^{\hat{s}}\left(\mathrm{x}_{T_{1}^{S}} \in \widehat{V_{1}}, T_{1}^{S} \in T\right) .
\end{aligned}
$$

Claim 2: For $t_{1}>0$ and $s_{1} \in S_{+}$,

$$
\mathbf{P}^{\widehat{s_{1}}}\left(\mathrm{X}_{t_{1}} \in A, t_{1}<T^{S}\right) \leq \mathbf{P}^{s_{1}}\left(\mathrm{X}_{t_{1}} \in A^{*}, t_{1}<T^{S}\right) .
$$

Claim 3: For $t_{1}>0$ and $s_{1} \in S_{+}$,

$$
\begin{aligned}
& \mathbf{P}^{s_{1}}\left(\mathrm{X}_{t_{1}} \in A, t_{1}<T^{S}\right)+\mathbf{P}^{\widehat{s_{1}}}\left(\mathrm{x}_{t_{1}} \in A, t_{1}<T^{S}\right) \\
& =\mathbf{P}^{s_{1}}\left(\mathrm{X}_{t_{1}} \in A^{*}, t_{1}<T^{S}\right)+\mathbf{P}^{\widehat{s_{1}}}\left(\mathrm{X}_{t_{1}} \in A^{*}, t_{1}<T^{S}\right) .
\end{aligned}
$$

Claim 4: For $v \in V, S_{1} \subset S_{+}, T \subset(0, \infty)$,

$$
\mathbf{P}^{v}\left(\mathrm{x}_{T_{1}^{V}} \in S_{1}, T_{1}^{V} \in T\right) \leq \mathbf{P}^{v}\left(\mathrm{x}_{T_{1}^{V}} \in \widehat{S_{1}}, T_{1}^{V} \in T\right) .
$$

Claim 5: For $v \in V, S_{1} \subset S_{+}, T \subset(0, \infty)$,

$$
\mathbf{P}^{\hat{v}}\left(\mathrm{X}_{T_{1}^{\widehat{V}}} \in \widehat{S_{1}}, T_{1}^{\widehat{V}} \in T\right) \leq \mathbf{P}^{\hat{v}}\left(\mathrm{X}_{T_{1}^{\widehat{V}}} \in S_{1}, T_{1}^{\widehat{V}} \in T\right) .
$$

Claim 6: For $v \in V, S_{1} \subset S_{+}, T \subset(0, \infty)$,

$$
\mathbf{P}^{v}\left(\mathrm{X}_{T_{1}^{V}} \in S_{1}, T_{1}^{V} \in T\right)=\mathbf{P}^{\hat{v}}\left(\mathrm{x}_{T_{1}^{\widehat{V}}} \in \widehat{S_{1}}, T_{1}^{\widehat{V}} \in T\right) .
$$


Claims 1 and 6 follow from the reflection invariance of the symmetric stable process (see [16] $\S 10.27$, Theorem 10.14]). Claims 2 and 3 follow from Lemma 5 and the definition of the polarization $A^{*}$ of $A$. Claim 5 is equivalent to Claim 4 because of reflection invariance. Finally, Claim 4 is a consequence of Lemma 8 So (3.2) has been proved.

An inequality like (3.2) holds for every appropriate pair of bracket sets. The proof uses induction on the number $k$ of $S$ 's in the bracket sets. We will only sketch this proof, because it is similar to the proof of (3.2). First suppose that $k=2$. Then the corresponding bracket set of $\mathcal{A}_{s}$ is of the form $[S \ldots S]$, where only the letters $U$ and $V$ appear (alternatively) between the two $S^{\prime}$ 's. The proof of

$$
\mathbf{P}^{s}([S \ldots S])+\mathbf{P}^{\hat{s}}([S \ldots S]) \leq \mathbf{P}^{s}\left([S \ldots S]^{*}\right)+\mathbf{P}^{\hat{s}}\left([S \ldots S]^{*}\right)
$$

in this case is similar to the proof of (3.2): one writes formulae (using Lemma 9 iteratively) for the left and right hand sides of (3.16) and compares the corresponding terms using Claims 1-6. For $k \geq 3$, the pertinent bracket set of $\mathcal{A}_{s}$ has the form $[S \ldots S \ldots S]$, where between the first and second $S$ there exist only the letters $U$ and $V$, and between the second and the last $S$ there exist some $U$ 's, some $V$ 's, and $k-3 S$ 's. Again by using Lemma 9 iteratively, we are reduced to the case of bracket sets with $k-1 S^{\prime}$ 's, and therefore, by the inductive assumption, we are done.

Since $\mathcal{A}_{s}$ and $\mathcal{A}_{s}^{*}$ are the unions of their bracket sets, (3.1) has been proved, and therefore (1.27) is also proved (under Assumptions A,B,C).

We now remove Assumption C; we continue to make Assumptions A and B. We denote the interior of a set $E$ in $\mathbb{R}^{n}$ by $\breve{E}$. We decompose the set $A$ as follows:

$$
\begin{aligned}
A & =(A \cap S) \cup(A \cap \breve{U}) \cup(A \cap \breve{V}) \cup(A \cap U \cap \partial S) \cup(A \cap V \cap \partial S) \\
& =: \quad A_{1} \cup A_{2} \cup A_{3} \cup A_{4} \cup A_{5} .
\end{aligned}
$$

By Assumption B, $m_{n}(\partial S)=0$. Since the measure $P_{t}(s, \cdot)$ is absolutely continuous with respect to $m_{n}$, for every $s$,

$$
P_{t}^{D}\left(s, A_{4}\right)=P_{t}^{D}\left(s, A_{5}\right)=P_{t}^{D^{*}}\left(s, A_{4}^{*}\right)=P_{t}^{D^{*}}\left(s, A_{5}^{*}\right)=0 .
$$

So we have to show that for $k=1,2,3$,

$$
P_{t}^{D}\left(s, A_{k}\right)+P_{t}^{D}\left(\hat{s}, A_{k}\right) \leq P_{t}^{D^{*}}\left(s, A_{k}^{*}\right)+P_{t}^{D^{*}}\left(\hat{s}, A_{k}^{*}\right) .
$$

We will prove (3.17) for $k=1$; the proof for $k=2,3$ is similar. So we assume that $A \subset S$. We also make for the moment the following additional assumption:

Assumption D: $\bar{A} \subset S$.

We consider three sequences $\left\{S_{j}\right\},\left\{U_{j}\right\},\left\{V_{j}\right\} j=1,2,3, \ldots$, of open sets with the following properties (the construction of these sequences is standard):

(i) $\overline{S_{j}} \cap \overline{U_{j}}=\emptyset, \overline{S_{j}} \cap \overline{V_{j}}=\emptyset, \overline{U_{j}} \cap \overline{V_{j}}=\emptyset$.

(ii) $S_{j} \subset \overline{S_{j}} \subset S_{j+1}, U_{j} \subset \overline{U_{j}} \subset U_{j+1}, V_{j} \subset \overline{V_{j}} \subset V_{j+1}$.

(iii) $\bigcup_{j=1}^{\infty} S_{j}=S, \bigcup_{j=1}^{\infty} U_{j}=U, \bigcup_{j=1}^{\infty} V_{j}=V$.

(iv) Each of $S_{j}, U_{j}, V_{j}$ is the union of a finite number of cubes.

By Assumption $D, A \subset S_{j}$ for all sufficiently large $j$. Let $D_{j}:=S_{j} \cup U_{j} \cup V_{j}$ and observe that $D_{j} \uparrow D$ and $D_{j}^{*} \uparrow D^{*}$. By the first part of the proof,

$$
P_{t}^{D_{j}}(s, A)+P_{t}^{D_{j}}(\hat{s}, A) \leq P_{t}^{D_{j}^{*}}\left(s, A^{*}\right)+P_{t}^{D_{j}^{*}}\left(\hat{s}, A^{*}\right),
$$

for $s \in S$ and all sufficiently large $j$. Taking limits in (3.18) and using Lemma 3 we obtain

$$
P_{t}^{D}(s, A)+P_{t}^{D}(\hat{s}, A) \leq P_{t}^{D^{*}}\left(s, A^{*}\right)+P_{t}^{D^{*}}\left(\hat{s}, A^{*}\right) .
$$


To remove Assumption $\mathrm{D}$, take an increasing sequence $A_{j}$ with $\overline{A_{j}} \subset S$ and $A_{j} \uparrow A$. Then (3.19) holds with $A_{j}$ in place of $A$, and, taking limits as $j \rightarrow \infty$, we conclude that (3.19) holds even if $A$ does not satisfy Assumption D.

We next remove Assumption B; we continue to make the Assumption A (that $A$ is bounded). Take a sequence of open sets $D_{j}$ such that $D_{j} \uparrow D$ and $D_{j}$ is the union of a finite number of cubes. Since $A$ is bounded, $A \subset D_{j}$ for all sufficiently large $j$. Then, by what we have proved so far, (3.18) holds, and taking limits, we see that (3.19) holds too.

Finally, we remove Assumption A. Take a sequence $A_{j}$ of bounded Borel sets with $A_{j} \uparrow A$. Then, by what we have proved so far,

$$
P_{t}^{D}\left(s, A_{j}\right)+P_{t}^{D}\left(\hat{s}, A_{j}\right) \leq P_{t}^{D^{*}}\left(s, A_{j}^{*}\right)+P_{t}^{D^{*}}\left(\hat{s}, A_{j}^{*}\right) .
$$

We take limits as $j \rightarrow \infty$ and obtain

$$
P_{t}^{D}(s, A)+P_{t}^{D}(\hat{s}, A) \leq P_{t}^{D^{*}}\left(s, A^{*}\right)+P_{t}^{D^{*}}\left(\hat{s}, A^{*}\right) .
$$

The proof of Theorem 4 is now complete.

\section{Proof of Theorems 1,2 , and 3}

4.1. Proof of Theorem 3. The inequalities (1.15), (1.16), and (1.17) for the Green function follow from Theorem 4 and the identity (2.5). Next we prove (1.21). Let $s \in D \cap \widehat{D}$ and $y \in D^{c}$ with $y^{*} \in\left(D^{*}\right)^{c}$. We consider two cases. Case 1: $y \in \mathbb{R}_{-}^{n}$ and Case 2: $y \in \mathbb{R}_{+}^{n}$. We deal now with Case 1 . In this case, we have $y^{*}=\hat{y}$. In the following calculations we use the symmetry of the Green function, the inequalities (1.16), (1.17), the identity (2.6) and the obvious inequality $|z-y| \geq|z-\hat{y}|, z \in S_{+}$. We start with the left hand side of (1.21):

$$
\begin{aligned}
K^{D}(s, y)+K^{D}(\hat{s}, y) & =\mathcal{A}(n, \alpha) \int_{D} \frac{G^{D}(s, z)+G^{D}(\hat{s}, z)}{|z-y|^{n+\alpha}} d z \\
& =\mathcal{A}(n, \alpha)\left[\int_{S}+\int_{U}+\int_{V}\right]=: \mathcal{A}(n, \alpha)\left(J_{S}+J_{U}+J_{V}\right) .
\end{aligned}
$$

Similarly,

$$
\begin{aligned}
& K^{D^{*}}(s, \hat{y})+K^{D^{*}}(\hat{s}, \hat{y})=\mathcal{A}(n, \alpha) \int_{D^{*}} \frac{G^{D^{*}}(s, z)+G^{D^{*}}(\hat{s}, z)}{|z-\hat{y}|^{n+\alpha}} d z \\
&=\mathcal{A}(n, \alpha)\left[\int_{S}+\int_{U}+\int_{\widehat{V}}\right]=: \mathcal{A}(n, \alpha)\left(J_{S}^{*}+J_{U}^{*}+J_{V}^{*}\right) .
\end{aligned}
$$

We will prove that $J_{S} \leq J_{S}^{*}, J_{U} \leq J_{U}^{*}, J_{V} \leq J_{V}^{*}$. For the integral $J_{S}$ we have

$$
J_{S}=\int_{S_{+}}\left[\frac{G^{D}(s, z)+G^{D}(\hat{s}, z)}{|z-y|^{n+\alpha}}+\frac{G^{D}(s, \hat{z})+G^{D}(\hat{s}, \hat{z})}{|\hat{z}-y|^{n+\alpha}}\right] d z .
$$

Similarly, for the integral $J_{S}^{*}$ we have

$$
J_{S}^{*}=\int_{S_{+}}\left[\frac{G^{D^{*}}(s, z)+G^{D^{*}}(\hat{s}, z)}{|z-\hat{y}|^{n+\alpha}}+\frac{G^{D^{*}}(s, \hat{z})+G^{D^{*}}(\hat{s}, \hat{z})}{|\hat{z}-\hat{y}|^{n+\alpha}}\right] d z .
$$


Therefore, it suffices to prove the following inequality for $s, z \in S_{+}$:

$$
\begin{aligned}
\frac{G^{D}(s, z)+G^{D}(\hat{s}, z)}{|z-y|^{n+\alpha}}+ & \frac{G^{D}(s, \hat{z})+G^{D}(\hat{s}, \hat{z})}{|\hat{z}-y|^{n+\alpha}} \\
& \leq \frac{G^{D^{*}}(s, z)+G^{D^{*}}(\hat{s}, z)}{|z-\hat{y}|^{n+\alpha}}+\frac{G^{D^{*}}(s, \hat{z})+G^{D^{*}}(\hat{s}, \hat{z})}{|\hat{z}-\hat{y}|^{n+\alpha}}
\end{aligned}
$$

This inequality follows from the following simple lemma applied with

$$
\begin{array}{cl}
A=G^{D}(s, z), \quad B=G^{D}(\hat{s}, z), & C=G^{D}(s, \hat{z}), \quad D=G^{D}(\hat{s}, \hat{z}), \\
A^{*}=G^{D^{*}}(s, z), \quad B^{*}=G^{D^{*}}(\hat{s}, z), & C^{*}=G^{D^{*}}(s, \hat{z}), \quad D^{*}=G^{D^{*}}(\hat{s}, \hat{z}), \\
E=|z-y|^{-n-\alpha}=|\hat{y}-\hat{s}|^{-n-\alpha}, & F=|z-\hat{y}|^{-n-\alpha}=|\hat{z}-y|^{-n-\alpha} .
\end{array}
$$

Lemma 10. Let $A, B, C, D, A^{*}, B^{*}, C^{*}, D^{*}, E, F$ be nonnegative numbers such that $A+B+C+D \leq A^{*}+B^{*}+C^{*}+D^{*}, C+D \leq A^{*}+B^{*}$, and $E \leq F$. Then $(A+B) E+(C+D) F \leq\left(A^{*}+B^{*}\right) F+\left(C^{*}+D^{*}\right) E$.

Proof. The desired inequality is equivalent to

$$
\begin{aligned}
(A+ & B+C+D) E+(C+D)(F-E) \\
& \leq\left(A^{*}+B^{*}+C^{*}+D^{*}\right) E+\left(A^{*}+B^{*}\right)(F-E),
\end{aligned}
$$

which is true.

The proof of the inequalities $J_{U} \leq J_{U}^{*}$ and $J_{V} \leq J_{V}^{*}$ is similar. So (1.21) is proved in Case 1. The proof for Case 2 is similar. The proof of $(1.20)$ is also similar.

The inequalities (1.18) and (1.19) follow from (1.20) and (1.21) and the fact that $K^{D}$ is the density of harmonic measure (see identity (1.12)). The proof of Theorem 3 is now complete.

4.2. Proof of Theorem 2, Let $K$ be a compact set in $\mathbb{R}^{n}$. By the fact that $\left(K^{c}\right)^{*}=\widehat{\left(K^{*}\right)^{c}}$ and by the identity (2.11) we obtain, for $x \in \Pi_{n-1} \cap K^{c}$,

$$
1-\mathbf{P}^{x}\left(T^{K^{c}}<\infty\right)=\lim _{|y| \rightarrow \infty} \mathcal{A}(n, \alpha)^{-1}|y|^{n-\alpha} G^{K^{c}}(x, y)
$$

and

$$
1-\mathbf{P}^{x}\left(T^{\left(K^{*}\right)^{c}}<\infty\right)=\lim _{|y| \rightarrow \infty} \mathcal{A}(n, \alpha)^{-1}|y|^{n-\alpha} G^{\left(K^{c}\right)^{*}}(x, y) .
$$

By (4.6), (4.7), and Theorem 3(a),

$$
\mathbf{P}^{x}\left(T^{\left(K^{*}\right)^{c}}<\infty\right) \leq \mathbf{P}^{x}\left(T^{K^{c}}<\infty\right), x \in \Pi_{n-1} \cap K^{c} .
$$

Now (4.8) and (2.10) yield

$$
C_{\alpha}\left(K^{*}\right) \leq C_{\alpha}(K)
$$

and Theorem 2 is proved. 
4.3. Proof of Theorem 1. In this proof we will use results and ideas taken from the articles [8] of F.Brock and A.Yu.Solynin, and [24] of J.Sarvas. We refer to these papers for the unexplained terminology we will use below.

Let $K$ be a compact set in $\mathbb{R}^{n}$ with $0<m_{n}(K)<\infty$. By Lemma 7.2 of [8], there exists a sequence of polarizations $\mathcal{P}_{j}, j=1,2, \ldots$ (with respect to suitable $(n-1)$-dimensional planes parallel to $\left.\Pi_{n-1}\right)$, such that

$$
\lim _{k \rightarrow \infty} \mathcal{Q}_{k}(K)=K^{\sharp},
$$

where $K^{\sharp}$ is the Steiner symmetrization of $K$ with respect to $\Pi_{n-1}, \mathcal{Q}_{k}=\mathcal{P}_{k}$ 。 $\mathcal{P}_{k-1}, k=2,3, \ldots$, and the convergence is in the Hausdorff metric.

Lemma 11. For every $r>0$, there exists a $k_{o} \in\{2,3, \ldots\}$ such that $K^{\sharp} \subset$ $\mathcal{Q}_{k}\left(K+r \bar{B}^{n}\right)$ for all $k \geq k_{o}$, where $\bar{B}^{n}$ is the closure of the unit ball in $\mathbb{R}^{n}$ centered at the origin.

Proof. Let $r>0$. By 4.10), we can choose $k_{o}$ such that

$$
K^{\sharp} \subset \mathcal{Q}_{k}(K)+r \bar{B}^{n}
$$

for all $k \geq k_{o}$. Since polarization is a smoothing transformation (see [1, p. 58], [8, Lemma 5.1]),

$$
\mathcal{Q}_{k}(K)+r \bar{B}^{n} \subset \mathcal{Q}_{k}\left(K+r \bar{B}^{n}\right) .
$$

The next lemma presents a well-known property of capacity; see [12].

Lemma 12. Let $\left\{K_{j}\right\}$ be a decreasing sequence of compact sets with $\bigcap_{j=1}^{\infty} K_{j}=K$. Then

$$
\lim _{j \rightarrow \infty} C_{\alpha}\left(K_{j}\right)=C_{\alpha}(K)
$$

Lemma 13.

$$
C_{\alpha}(K) \geq C_{\alpha}\left(K^{\sharp}\right) .
$$

Proof. Let $K_{j}:=K+\frac{1}{j} \bar{B}^{n}$ and $\varepsilon>0$. The sequence $\left\{K_{j}\right\}$ is decreasing, and $\bigcap_{j=1}^{\infty} K_{j}=K$. By Lemma 12, there exists a $j_{o}$ such that

$$
C_{\alpha}(K)+\varepsilon \geq C_{\alpha}\left(K_{j_{o}}\right)=C_{\alpha}\left(K+\frac{1}{j_{o}} \bar{B}^{n}\right) .
$$

By Lemma 11] we can choose a $k_{o}$ such that

$$
K^{\sharp} \subset \mathcal{Q}_{k_{o}}\left(K+\frac{1}{j_{o}} \bar{B}^{n}\right),
$$

and the monotonicity of $\alpha$-capacity (see [18, p.140]) yields

$$
C_{\alpha}\left(K^{\sharp}\right) \leq C_{\alpha}\left(\mathcal{Q}_{k_{o}}\left(K+\frac{1}{j_{o}} \bar{B}^{n}\right)\right) .
$$

Finally, Theorem 2, (4.13), and (4.14) give

$$
C_{\alpha}(K)+\varepsilon \geq C_{\alpha}\left(K+\frac{1}{j_{o}} \bar{B}^{n}\right) \geq C_{\alpha}\left(\mathcal{Q}_{k_{o}}\left(K+\frac{1}{j_{o}} \bar{B}^{n}\right)\right) \geq C_{\alpha}\left(K^{\sharp}\right) .
$$

Since $\varepsilon$ is arbitrary, Lemma 13 is proved. 
The Steiner symmetrization $K^{\sharp}$ of $K$ with respect to the $(n-1)$-dimensional plane $\Pi_{n-1}$ is a 1-dimensional Steiner symmetrization (in the terminology of [24]). By a fundamental theorem in the theory of symmetrization [24, Theorem 4.32], every $k$-dimensional Steiner symmetrization $(2 \leq k \leq n)$ can be approximated (in the Hausdorff metric) by a sequence of successive $(k-1)$-dimensional Steiner symmetrizations. This fact combined with Lemma 13, and an argument similar to that in the proof of Lemma 13, show that

$$
C_{\alpha}(K) \geq C_{\alpha}(\operatorname{Sym} K),
$$

where Sym denotes any $k$-dimensional Steiner symmetrization $(1 \leq k \leq n)$. In particular, for $k=n$, $\operatorname{Sym} K$ is a ball $\mathbb{B}_{V}$ with $m_{n}\left(\mathbb{B}_{V}\right)=m_{n}(K)=V$. Therefore (4.16) implies

$$
C_{\alpha}(K) \geq C_{\alpha}\left(\mathbb{B}_{V}\right)
$$

and Theorem 1 is proved.

\section{FURTHER RESUlts}

5.1. Some more consequences of Theorem 4 . Theorem 4 implies that

$$
p_{t}^{D}(x, y) \leq p_{t}^{D^{*}}\left(x^{*}, y^{*}\right),
$$

for all $t>0$ and $x, y \in D$. It follows from (5.1) that

$$
\int_{D} p_{t}^{D}(x, y) d y \leq \int_{D^{*}} p_{t}^{D^{*}}\left(x^{*}, y\right) d y,
$$

for all $x \in D$ and $t>0$. The inequality (5.2) is equivalent to

$$
\mathbf{P}^{x}\left(T^{D}>t\right) \leq \mathbf{P}^{x^{*}}\left(T^{D^{*}}>t\right), t>0,
$$

which implies that

$$
\mathbf{E}^{x}\left[\phi\left(T^{D}\right)\right] \leq \mathbf{E}^{x^{*}}\left[\phi\left(T^{D^{*}}\right)\right],
$$

for all nonnegative increasing functions $\phi$.

Another consequence of (5.3) is the following ( $D$ is assumed to be bounded for this result): Let $\lambda_{D}=\lambda_{D, \alpha}$ denote the first Dirichlet eigenvalue for the operator associated to the process $\mathrm{X}_{t}$ in $D$; see [2], [9]. Then

$$
-\lambda_{D}=\lim _{t \rightarrow \infty} \frac{1}{t} \log \mathbf{P}^{x}\left(T^{D}>t\right) .
$$

So it follows from (15.3) that

$$
\lambda_{D} \geq \lambda_{D^{*}}
$$

One can now use the approach to symmetrization via polarization to prove similar eigenvalue estimates for various types of symmetrization. In particular, one can prove

$$
\lambda_{D} \geq \lambda_{\mathbb{B}},
$$

where $\mathbb{B}$ is the ball in $\mathbb{R}^{n}$ with $m_{n}(\mathbb{B})=m_{n}(D)$, centered at the origin. This result has been proved (by a different method) in [2]. For Brownian motion (i.e., for $\alpha=2)$, (5.7) is the classical Rayleigh-Faber-Krahn inequality. 
5.2. On a problem of Mattila. In this subsection we assume for simplicity that $n=2$ and $\alpha=1$. Let $K$ be a compact set in the plane. By $p_{\theta}(K), \theta \in[0, \pi)$, we denote the orthogonal projection of $K$ on the line through the origin in $\mathbb{R}^{2}$ forming an angle $\theta$ with the positive $x$-axis. The length (1-dimensional Hausdorff measure) of $p_{\theta}(K)$ is denoted by $\left|p_{\theta}(K)\right|$. The following sharp inequality has been proved by Mattila 19:

$$
\pi^{2} \mathcal{A}(2,1) C_{1}(K) \leq \int_{0}^{\pi}\left|p_{\theta}(K)\right| d \theta
$$

Equality holds for disks. On the other hand, by Theorem 1, we have

$$
C_{1}(K) \geq C_{1}(\mathbb{B}),
$$

where $\mathbb{B}$ is the disk centered at the origin with radius $r:=\sqrt{m_{2}(K) / \pi}$. The 1 capacity of the disk of radius $r$ has been computed by N.S.Landkof [18, p.163]:

$$
C_{1}(\mathbb{B})=\frac{2}{\pi \mathcal{A}(2,1)} r=\frac{2}{\pi^{3 / 2} \mathcal{A}(2,1)} \sqrt{m_{2}(K)} .
$$

Combining (5.8), (5.9), and (5.10), we obtain the following sharp inequality:

$$
\sqrt{m_{2}(K)} \leq \frac{1}{2 \sqrt{\pi}} \int_{0}^{\pi}\left|p_{\theta}(K)\right| d \theta .
$$

Equality holds for disks. This result gives a partial answer to a problem raised by Mattila [19, p.192].

\section{ACKNOWLEDGEMENTS}

My interest in symmetric stable processes started up when I attended a talk by Jang-Mei Wu during the Nevanlinna Colloquium (Helsinki, August 2000). I thank her for the advice and instructions she gave me after her talk; I also thank Matti Vuorinen who invited me to the Nevanlinna Colloquium. I express my gratitude to Al Baernstein for his suggestions and for bringing my attention to the article [19]. I also thank Vladimir Dubinin for his encouragement and Dimitri Gatzouras for helpful discussions. I especially thank the referee for his/her corrections and suggestions.

\section{REFERENCES}

1. A. Baernstein II, A unified approach to symmetrization, in Partial Differential Equations of Elliptic type (Cortona 1992) pp.47-91, Cambridge Univ. Press, 1994. MR 96e:26019

2. R. Bañuelos, R. Latala, and P. J. Méndez-Hernández, A Brascamp-Lieb-Luttinger-type inequality and applications to symmetric stable processes. Proc. Amer. Math. Soc. 129 (2001), 2997-3008. MR 2002c:60125

3. D. Betsakos, Polarization, conformal invariants, and Brownian motion. Ann. Acad. Sci. Fenn. Ser. A I Math. 23 (1998), 59-82. MR 99g:31004

4. R. M. Blumenthal and R. K. Getoor, Some theorems on stable processes. Trans. Amer. Math. Soc. 95 (1960), 263-273. MR 22:10013

5. R. M. Blumenthal and R. K. Getoor, Markov Processes and Potential Theory. Academic Press, 1968. MR 41:9348

6. K. Bogdan, The boundary Harnack principle for the fractional Laplacian. Studia Math. 123 (1997), 43-80. MR 98g:31005

7. K. Bogdan and T. Byczkowski, Potential theory for the $\alpha$-stable Schrödinger operator on bounded Lipschitz domains. Studia Math. 133 (1999), 53-92. MR 99m:31010 
8. F. Brock and A. Yu. Solynin, An approach to symmetrization via polarization. Trans. Amer. Math. Soc. 352 (2000), 1759-1796. MR 2001a:26014

9. Z. Q. Chen and R. Song, Intrinsic ultracontractivity and conditional gauge for symmetric stable processes. J. Funct. Anal. 150 (1997), 204-239. MR 98j:60103

10. Z. Q. Chen and R. Song, Estimates on Green functions and Poisson kernels for symmetric stable processes. Math. Ann. 312 (1998), 465-501. MR 2000b:60179

11. Z. Q. Chen and R. Song, Martin boundary and integral representation for harmonic functions of symmetric stable processes. J. Funct. Anal. 159 (1998), 267-294. MR 2000d:60128

12. K. L. Chung, Lectures from Markov Processes to Brownian Motion. Springer-Verlag, 1982. MR 84c:60091

13. K. L. Chung and M. Rao, Equilibrium and energy. Probab. Math. Statist. 1 (1980), 99-108. MR 83f: 60102

14. V. N. Dubinin, Capacities and geometric transformations of subsets in n-space. Geometric and Functional Anal. 3 (1993), 342-369. MR 94f:31008

15. V. N. Dubinin, Symmetrization in the geometric theory of functions of a complex variable. Russian Math. Surveys 49 (1994), no. 1, 1-79. MR 96b:30054

16. E. B. Dynkin, Markov Processes, Volume I. Springer 1965. MR 33:1887

17. E. B. Dynkin, Markov Processes, Volume II. Springer 1965. MR 33:1887

18. N. S. Landkof, Foundations of Modern Potential Theory. Springer-Verlag, 1972. MR 50:2520

19. P. Mattila, Orthogonal projections, Riesz capacities, and Minkowski content. Indiana Univ. Math. J. 39 (1990), 185-198. MR 91d:28018

20. G. Pólya and G. Szegö, Isoperimetric Inequalities in Mathematical Physics. Princeton Univ. Press, 1951. MR 13:270d

21. S. C. Port, On hitting places for stable processes. Ann. Math. Statist. 38 (1967), 1021-1026. MR 35:5002

22. S. C. Port, A remark on hitting places for transient stable process. Ann. Math. Statist. 39 (1968), 365-371. MR 37:980

23. S. C. Port and C. J. Stone, Brownian Motion and Classical Potential Theory. Academic Press, 1978. MR 58:11459

24. J. Sarvas, Symmetrization of condensers in $n$-space. Ann. Acad. Sci. Fenn. Ser. A I No. 522 (1972), 1-44. MR 50:606

25. A. Yu. Solynin, Functional inequalities via polarization. Algebra i Analiz 8 (1996), 148-185 (in Russian); English transl. in St. Petersburg Math. J. 8 (1997), 1015-1038. MR 98e:30001a

26. R. Song and J.-M. Wu, Boundary Harnack principle for symmetric stable processes. J. Funct. Anal. 168 (1999), 403-427. MR 2001b:60092

27. G. Szegö, Über einige Extremalaufgaben der Potentialtheorie, Math. Zeitschrift 31 (1930), 583-593.

28. V. Wolontis, Properties of conformal invariants. Amer. J. of Math. 74 (1952), 587-606. MR $14: 36 \mathrm{c}$

Department of Mathematics, Aristotle University of Thessaloniki, 54124 ThessaLONIKI, GREECE

E-mail address: betsakos@auth.gr 